\title{
Proprioceptive space perception after anaesthetization of the mid-interphalangeal joint of the finger
}

\author{
JOHN K. COLLINS \\ Trent University, Peterborough, Ontario, Canada
}

\begin{abstract}
The effect of a digital block on the voluntary positioning of the index finger from a point of extreme extension through $45^{\circ}$ flexion was examined in 12 subjects. A xylocaine ring block of the second proximal interphalangeal joint was used to eliminate the joint receptors while weights were attached to the finger. An inverse relationship was found between weight and distance moved when the subject was required to move the index finger of the nonpreferred hand to the required position. The results indicate that the muscular system is ancillary to the joint system in proprioceptive space perception.
\end{abstract}

The observation of the proprioceptive spatial aftereffect (Collins, 1971) suggested that afferent impulses from the muscles are employed in the monitoring of performance and the positioning of the limb in space. Since Goldscheider (1898) proposed that the joints contributed more than the muscles to the perception of movement, two different paradigms have been used to study the kinestheticproprioceptive modality. The contradictory reports concerning whether or not muscle afferents are involved in space perception (Browne, Lee, \& Ring, 1954; Lloyd \& Caldwell, 1965; Mountcastle \& Powell, 1959; Provins, 1958) may have arisen from a confusion in interpreting the data collected by these methods. The first is concerned with the perception of the limb in space once it has been positioned, either voluntarily or involuntarily, and the second is concerned with the thresholds of movement. It is suggested, in the present paper, that to test the proposition that the sense of position and movement of the limb depend solely on the receptor elements in the joints (Mountcastle, 1957; Mountcastle \& Powell, 1959; Rose \& Mountcastle, 1959), a third paradigm should be used: that which is concerned with the voluntary movement of the limb to a specified position in space.

The dual process of both the receptor elements in the muscles and joints supplying information concerning the resolution of the positioning of the limb is consistent with the theory of space perception outlined by Day (Note 1).

In relation to light and sound, he argued "... because of the transmission characteristics of light and sound energy in physical space, there are invariant relationships between certain properties of the energy. In consequence of these physical invariances there occur identities of stimulation at the sensory surfaces for a multiplicity of different physical arrangements" (Day, Note 1, p. 2). In order to resolve the identity of relationships between size and distance, which result in the same retinal projection, information for distance must be available. Similarly, information must be available for the perception of one of the components in order to resolve the identities of the relationships between the retinal projection and different shapes at different orientations, and for different bodily and object orientations. The theory applies equally well to other sense modalities, such as head rotation and location of sound source, and the external tactile stimulation achieved by movement of an object along the surface of the skin or movement of the limb across a stationary object.

It was within this theoretical framework that the present experiment was designed. It is necessary to make three points of explanation. First, the limb which is moved by a joint system is of a constant mass. Second, the work done by a muscle is a function of the mass moved and the distance it is moved. Third, judgments of the position of the limb are mediated by the joint receptors. The question that has been studied since the beginning of the century has not been concerned with whether or not the joints mediate positional judgments: this can be taken as a statement of fact. The problem being considered is whether or not there is a second ancillary system which helps the successful monitoring of performance.

Generally, since the mass to be moved is a constant, the work done will vary directly with the distance moved. Further, there will be an infinite number of invariant relations between the work done by the muscles $(w)$ and the distance traveled by the limb (d), that is, $w_{1} / d_{1}=w_{2} / d_{2}=w_{3} / d_{3}$. This model, in its simplest form, assumes time and acceleration are constant.

If the joints supply information regarding the distance moved, then the muscles could supply additional information by way of the work done to move 
the limb. Such an explanation could help resolve the controversy that has arisen from the results of experiments using active and passive movement during stimulation of the limb. The muscles must be actively stimulated in order to supply positional information. If no work is done, no information is supplied. Conversely, when no information regarding the distance moved by the limb is available from the joints, such as when they are anesthetized, then information can only be gained by active movement of the limbs. Finally, since the work done by the muscles varies directly with the mass to be moved, then when no information regarding either the mass or the distance is available, constancy of perception should break down. Such a situation is analogous to the breakdown of size constancy when no information regarding size or distance is available and the judgment has to be made in terms of the retinal projection. The prediction in the present investigation was based on the assumption that a subject has a general conception of the work done by the muscles in moving the limb (in this case, the finger) through a certain distance in space. Since the distance was fixed and the mass of the limb was constant, the work done by the muscle (the cue used in the judgment) was of a certain fixed value. If the mass to be moved was then varied and the primary cues to distance were eliminated by anesthetization, the judgment of distance based on the constant $w$ should vary inversely with the mass moved.

\section{METHOD}

\section{Apparatus}

Basically, the apparatus consisted of three parts: a hand-clamp, a splint and weight tray, and the recording apparatus. The hand-clamp, which held the hand on a desk with the palmar surface upwards, was made of padded timber. Three grooves in the timber, which had a hinge joint at one end, held the middle, ring, and little finger tightly in place, while a metal rod secured the proximal joint of the index finger when a wing nut was tightened. A small brass disk, $2.5 \mathrm{~cm}$ in diameter, attached to a brass rod, $1.0 \mathrm{~cm}$ long, acted as a weight carrying tray. To this was attached a $5.0 \times 1.5 \mathrm{~cm}$ Perspex splint by a fine nylon line. The total weight of the carrying tray and splint was $2 \mathrm{~g}$. The nylon line from the splint to the tray passed through the grooved edge of a pulley wheel, $3 \mathrm{~cm}$ in diameter, which was attached to an Ohmag (Swiss) microtorque potentiometer. The impulses from the potentiometer were fed into a Both driver amplifier, and from there into a single-channel pen recorder moving at a speed of $5 \mathrm{~mm} / \mathrm{sec}$.

\section{Subjects \\ There were 12 subjects recruited from an introductory course in psychology. Although they were necessarily aware that the general aim of the experiment was to examine the effects of anesthetizing the joints on spatial judgments, none of them was aware of the specific predictions.}

\section{Procedure}

The task required of the subject was to move the midinterphalangeal joint of the index finger of the nonpreferred hand with weights attached, from the point of extreme extension through an angle of $45^{\circ}$. Weights of $3,6,9,12$, and $15 \mathrm{~g}$ were selected after it was ascertained by amputation of the index fingers of . cadavers between the fingertip and the midinterphalangeal joint that the mass usually moved was approximately $7.5 \mathrm{~g}$. These weights were within the limits set by the amount of work done by the muscles in voluntarily moving the finger through the full range of movement. Too heavy a weight would constitute an abnormal amount of strain on the finger and perhaps could be recognized as outside the range of the identity of relationships between work done and distance moved. At the other extreme, a range of weights which was too small could be below the differential threshold level of recognition.

During experimentation, each session was divided into two parts. Half the subjects attended the anesthetized session first, while the other half attended the control session first. The only difference between the two sessions was that one involved anesthetization. For the experimental session involving anesthesia, the fingers were injected with approximately $2-5 \mathrm{ml}$ of xylocaine $2 \%$ (lingocaine $\mathrm{HCl}$ ). Three skin punctures were made on either side of the finger distal to the metacarpophalangeal joint so that it would disperse subcutaneously about the interphalangeal joint. Sensibility to touch, prick, and pressure was tested 20-30 min after infiltration to satisfy that a digital block had been achieved. While there is always some doubt about the extent of anesthetization using this technique, it is generally accepted that information from the various receptors within the joints is blocked while the muscle spindles and neurotendinous end organs remain unaffected (Browne et al., 1954; Goodwin, McCloskey, \& Matthews, 1972a, b; Provins, 1958).

During the testing session, the subject was blindfolded by tight-fitting opaque goggles and the splint was taped across the distal interphalangeal joint to eliminate any other movement in the finger. The subject's hand was then placed in the handclamp with the palmar surface upwards. The only movement possible in the finger after it was secured was that of the midinterphalangeal joint. Following five practice judgments through an angle of $45^{\circ}$, the subjects were given one trial on each of the five conditions, moving the finger through the judged angle with the weights attached. The trials were presented in a different random order for each subject with a rest period of 1 min between each trial. A single trial under each condition was necessitated by the fact that, although the subjects were tested individually, they were given the anesthetizing agent in small groups. In order to eliminate the possibility of the affected joint recovering during a protracted session, a single trial on each condition was preferred.

\section{RESULTS AND DISCUSSION}

The mean positionings of the fingers, in degrees, under both normal and anesthetized conditions are presented in Figure 1. It can be seen that under normal conditions there was little variation in the positioning of the finger when the various weights were attached; however, after anesthetization, an inverse relationship was found between the distance moved and the mass attached to the finger. Greater intersubject variability was a feature of the positioning judgments under experimental conditions, but the trend in the mean judgments was unmistakable. Trend analysis showed that no significant variation had occurred in the mean judgments under normal conditions, $F(4,44)=0.91, \mathrm{p}>.05$, which is consistent with the constancy of perception of spatial judgments. However, analysis of the experimental data showed that a significant change occurred when 
the anesthetized judgments were made, $F(4,44)=3.66$, $p<.05$. When the sum of squares for trials was partitioned (Rodger, 1965), the linear component of the trend was found to be significant, $F(1,44)=$ $13.84, \mathrm{p}<.001$, whereas the residual component was not, $F(3,44)=0.26, \mathrm{p}>.05$. The relationship between the variables can therefore be represented by a straight line.

Two noticeable features regarding proprioceptive spatial judgments arise from the results. First, they are consistent with the hypothesis that the muscles, probably in conjunction with the tendon organs, perform an ancillary role in the perception of spatial judgments. This can be seen from the inverse relationship that exists between the mass which is moved by the muscles and the distance over which it is moved. The failure of the results to produce an exact and invariant relationship between work done and distance moved probably arises from the fact that the work done by the muscles is also a function of the acceleration of movement and the time taken to complete it. It could also be due to the proposition that when anesthetization such as employed in the present investigation is undertaken one can never be sure of the extent to which the member is anesthetized. Such a qualification must be placed upon the results of any experiment using this technique. The second noticeable effect was the

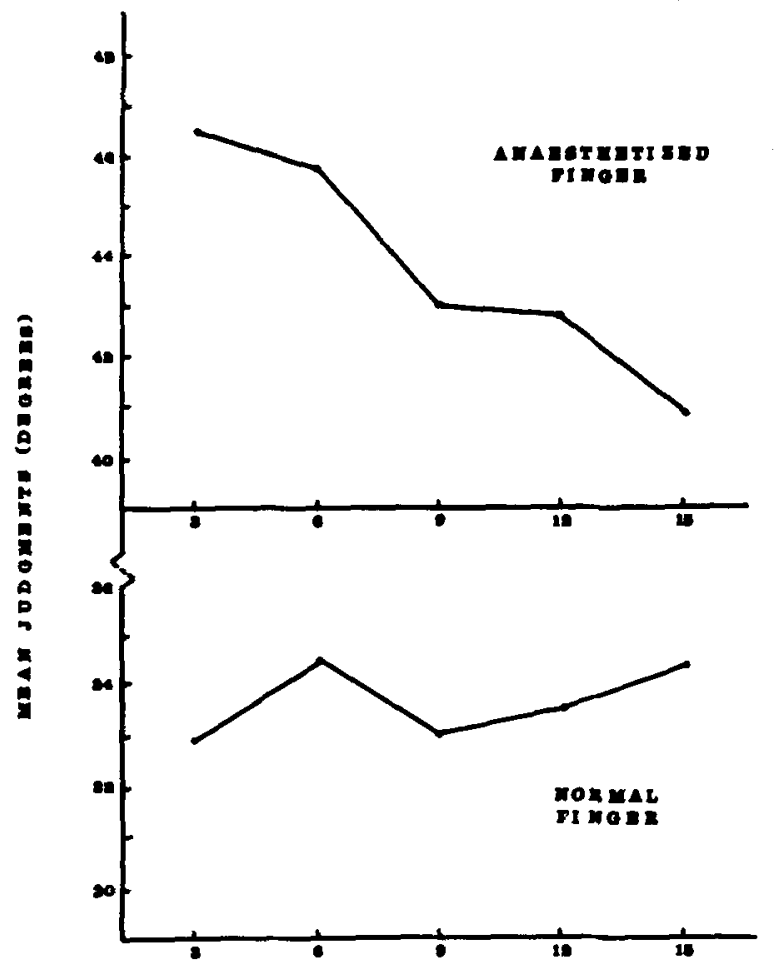

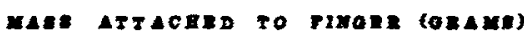

Figure 1. Mean finger positionings after the attachment of weights under normal and anesthetized conditions. distortion in the judgment of the position of the finger in space. In the control condition, the finger was moved through an angle of approximately $33^{\circ}$, which was perceived as $45^{\circ}$. It was not considered essential that perfect accuracy of positioning be obtained during practice trials. The consistency of the judgment was considered to be of greater importance, since it is known that joint sense in the fingers is relatively unreliable and crude (Merton, 1964). The notion of stability of judgments is consistent with the need to establish a mean pretest judgment in studies of aftereffects and illusions (Brown, 1953; Wertheimer, 1954). However, under experimental conditions, as the mass was increased, the angle through which the finger was moved was reduced from approximately $47^{\circ}$ to $40^{\circ}$. While the hypothesized decrement therefore occurred in the experimental group, the entire range of movement was greater than in the control condition. This discrepancy requires some explanation. It could be accounted for by the fact that the impulses from the joints, which usually signal the position of the finger, were blocked and the judgment which was asked for was an unfamiliar one. The combination of the reduced positional cues and the unfamiliar judgmental requirements could produce the results obtained. When the cues which usually signal the positioning of the limb are eliminated and the ancillary system takes over, it is not unreasonable to assume the limb would be moved initially through too great an angle because there are no other receptor elements which can signal the position of the limb. If the five practice judgments made prior to the addition of the weights to the finger are conceived of in terms of a motor learning situation of a new response (Collins \& Singer, 1968), this would tend to keep the response higher than the control level, and from this newly learned position the decremental effect resulting from the addition of weights could originate.

Part of the controversy, concerning the role of muscle receptors, can be resolved by considering active vs. passive procedures employed during experimentation. Studies using passive movement of the extremities following anesthesia (Browne et al., 1954; Gelfan \& Carter, 1967; Merton, 1964; Provins, 1958) seem to suggest that muscle spindle receptors are not responsible for any sense of passive movement, whether the paradigm calls for perception of the position of the limb in space or threshold of movement. Experiments such as those of Gelfan and Carter (1967), who artificially manipulated muscle length and tension by pulling on surgically exposed tendons, are considered to be employing passive movements, since the movements are not actively induced by the subject. However, different results are obtained when active movement is employed (Browne et al., 1954; Lloyd \& Caldwell, 1965; Merton, 1964). 
Merton (1964) suggested that there is no real sense of active movement, merely an inner consciousness of the effort required to move the limb, and that this inner consciousness is not a sensation dependent upon information from peripheral receptors. That is, a central sense of effort may be present in the absence of peripheral sensory information. His argument for the reintroduction of the sense of effort, which he suggests has been out of the vocabulary of the physiologist since Sherrington's (1900) time, could clear up some of the inconsistencies that have beclouded the whole issue of muscular involvement in space perception. Merton (1964) reported after anesthetization of the joint receptors in the thumb that, while the thumb remained insensitive to passive movement, active movement was unimpaired and made with much the same accuracy as before.

It is recognized that sense of effort may be misleading if movement is prevented, but it could prove to be of primary consideration in the voluntary positioning of the limb in space following anesthetization of joint receptors. The theoretical framework within which the present experiment was designed certainly fits in neatly with Merton's proposal, and the results become more plausible if the sense of effort hypothesis is invoked. Indeed, the whole issue arising from the differential results obtained from experiments requiring active or passive movement on the part of the subject can be resolved within the context of a central sense of effort mechanism.

The very complex signaling and feedback interactions of the afferent and efferent components involved among the fusimotor nerves, contractile sense organs, motoneurons, and tension receptors has yet to be discovered. The present findings, however, are consistent with a growing body of recent evidence (Collins, 1971; Goodwin et al., 1972a, b; Grigg, Finerman, \& Riley, 1973) that the proprioceptive (muscular) and kenesthetic (joint) systems are both involved somehow in the positioning of the limbs in space.

\section{REFERENCE NOTE}

1. Day, R. H. The identity of spatial illusions and spatial constancies. Paper presented at the Ninth Canberra Symposium on Perception, Monash Univeristy, Melbourne, 1966.

\section{REFERENCES}

Brown, K. T. Methodology for studying figural after-effects, and practice effects in the Müller-Lyer illusion. Amenican Journal of Psychology, 1953, 66, 629-634.

Browne, K., LeE, J., \& Ring, P. A. The sensation of passive movement of the metatarso-phalangeal joint of the great toe in man. Journal of Physiology, 1954, 126, 448-458.

Collins, J. K. Isolation of the muscular component in a proprioceptive spatial aftereffect. Journal of Experimental Psychology, 1971, 90, 297-299.

Collins, J. K., \& Singer, G. Interaction between sensory spatial aftereffects and persistence of response following behavioral compensation. Journal of Experimental Psycho$\log v, 1968,77,301-307$.

Gelfan, S., \& Carter, S. Muscle sense in man. Experimental Neurology, 1967, 18, 469-473.

GoldscheIder, A. Physiologie des Muskelsinnes. Leipzig: Barth. 1898.

Goodwin, G. M., McCloskey, D. I., \& Matthews, P. B. C. Proprioceptive illusions induced by muscle vibrations: Contribution by muscle spindles to perception? Science, 1972, 175, 1382-1384. (a)

Goodwin, G. M.. McCloskey, D. I., \& Matthews, P. B. C. The persistence of appreciable kinesthesia after paralysing joint afferents but preserving muscle afferents. Brain Research, 1972, 37, 326-329. (b)

Grigg, P., Finerman, G. A., \& Riley, L. H. Joint position sense after total hip replacement. Journal of Bone and Joint Surgery. 1973, 55, 1016-1025.

Lloyd, A, J., \& CAldwell, L. S. Accuracy of active and passive positioning of the leg on the basis of kinesthetic cues. Journal of Comparative and Physiological Psychology. $1965,60,102-106$.

Merton. P. A. Human position sense and sense of effort. Symposia of the Society for Experimental Biology. 1964, 18. 387.400 .

Mountcastle, V. B. Modality and topographic properties of single neurons of cat's somatic sensory cortex. Journal of Neurophysiology, 1957, 20, 408-434.

Mountcastle, V. B., \& Powell, T. P. S. Central neural mechanisms subserving position sense and kinesthesis. Bulletin of the John Hopkins Hospital, 1959, 105, 173-200.

Provins, K. A. The effect of peripheral nerve block on the appreciation and execution of finger movements. Journal of Physiology, 1958, 143, 55-67.

RODGER, R. S. Intermediate statistics. Sydney: Sydney University Bookshop, 1965.

Rose, J. E., \& Mountcastle, V. B. Touch and kinesthesis. In J. Field (Ed.), Handbook of physiology: 1. Neurophysiology Washington. D.C: American Physiological Society, 1959. Chap. 17.

Srerrington, C. S. The muscular sense. In E. A. Schafer (Ed.), Textbook of physiology, London: Pentland, 1900.

Wertheimer, $M$. Constant errors in the measurement of figural after-effects. American Joumal of Psychology, $1954,67,543-546$.

(Received for publication October 15, 1975; revision accepted March 22, 1976.) 